\title{
Autophagy and neurodegeneration
}

\author{
Rebecca A. Frake, Thomas Ricketts, Fiona M. Menzies, and David C. Rubinsztein \\ Department of Medical Genetics, Cambridge Institute for Medical Research, University of Cambridge School of Clinical Medicine, Cambridge, United Kingdom.
}

\begin{abstract}
Most neurodegenerative diseases that afflict humans are associated with the intracytoplasmic deposition of aggregate-prone proteins in neurons. Autophagy is a powerful process for removing such proteins. In this Review, we consider how certain neurodegenerative diseases may be associated with impaired autophagy and how this may affect pathology. We also discuss how autophagy induction may be a plausible therapeutic strategy for some conditions and review studies in various models that support this hypothesis. Finally, we briefly describe some of the signaling pathways that may be amenable to therapeutic targeting for these goals.
\end{abstract}

\section{Introduction}

Intracellular aggregates comprising misfolded proteins are a common feature of many neurodegenerative diseases. These aggregates contain different proteins, depending on the disease, and can be seen in different cell types and in different subcellular compartments. In some cases, mutations in a specific protein within the aggregates have been identified, such as $\alpha$-synuclein mutations in Parkinson's disease (PD) or expanded polyglutamine tracts in huntingtin in Huntington's disease (HD). In other cases the major protein species in the aggregates are not mutated. While these misfolded proteins may cause pathology via diverse mechanisms, in recent years there has been a focus on the role of autophagy in these diseases, both as a pathologic mechanism and as a therapeutic target.

The term autophagy describes a range of processes, including chaperone-mediated autophagy, microautophagy, and macroautophagy. Here we focus on macroautophagy, which we refer to as autophagy. In this process, cytoplasmic proteins and organelles are sequestered into autophagosomes and delivered to the lysosomes for degradation. The processes by which autophagosomes form are described in greater detail elsewhere (1). Briefly, autophagosomes form from the coalescence of membrane from sources including the plasma membrane, mitochondria, ER, and Golgi apparatus. Once formed, autophagosomes are trafficked to fuse with the lysosomes, forming autolysosomes; alternatively, they may fuse with endosomes to form amphisomes before fusing with lysosomes, where their contents are ultimately degraded (1).

In this Review we discuss the evidence that a disruption in autophagy might be a contributing factor in aggregate formation and the progression of neurodegenerative diseases. We detail the ever increasing list of neurodegenerative diseases in which autophagy perturbations have been reported and discuss a new class of diseases caused by mutations in core autophagy genes. We also discuss the ways in which macroautophagy may be upregulated to reduce levels of the toxic, aggregate-prone, intracytoplasmic

Authorship note: Rebecca A. Frake, Thomas Ricketts, and Fiona M. Menzies contributed equally to this work.

Conflict of interest: The authors have declared that no conflict of interest exists.

Reference information: J Clin Invest. 2015;125(1):65-74. doi:10.1172/JCI73944. proteins as a potential therapeutic strategy for these diseases. We highlight two major classes of autophagy-modulating drugs, which act either via mTOR inhibition or through mTOR-independent pathways, and outline recent studies investigating the effectiveness of these drugs in mouse models of neurodegenerative disease.

\section{Autophagy in the pathogenesis of neurodegenerative disease}

The importance of autophagy for the brain was highlighted by studies demonstrating that neuron-specific loss of core autophagy proteins (autophagy-related gene 7 [ATG7] and ATG5) in mice results in a neurodegenerative phenotype in the absence of any other contributing factors $(2,3)$. In particular, autophagy is required for maintenance of axonal homeostasis, and loss of autophagy results in axonal dystrophy (4). Autophagy is also a key regulator of the levels of intracytoplasmic, aggregate-prone proteins that cause neurodegenerative diseases, including polyglutamine-expanded huntingtin (HD) (5), mutant $\alpha$-synuclein (forms of PD) (6), mutant TDP-43 (ALS) (7), and wild-type and mutant tau (various dementias) (8). The clearance of such substrates is retarded when autophagy is compromised, and clearance is induced when autophagy is stimulated. Autophagic dysfunction has now been reported in a number of neurodegenerative diseases, which are outlined below and summarized in Figure 1.

Alzheimer's disease. Alzheimer's disease (AD) is characterized by extracellular amyloid- $\beta(\mathrm{A} \beta)$ plaques, which are generated through amyloid precursor protein (APP) cleavage, and neurofibrillary tangles, comprising paired helical filaments of intracellular, hyperphosphorylated tau, a microtubule-associated protein.

One of the first observations that suggested a role for altered autophagy in $\mathrm{AD}$ was the accumulation of autophagic vesicles in affected neurons $(9,10)$. While initially considered to represent increased autophagy, more recent evidence indicates that this accumulation is due to impaired autophagosome clearance. Presenilin-1 (PS1) is part of the $\gamma$-secretase complex required for $A \beta$ production; however, it also functions to facilitate $\mathrm{N}$-glycosylation of the $\mathrm{V}_{0} \mathrm{a}_{1}$ subunit of lysosomal vacuolar $\mathrm{H}^{+}$-ATPase (v-ATPase) and its trafficking to the lysosome to enable acidification of this organelle (11). PS1 and PS2 mutations cause familial autosomaldominant $\mathrm{AD}$ (12-14) and result in amyloid deposition, neuronal 


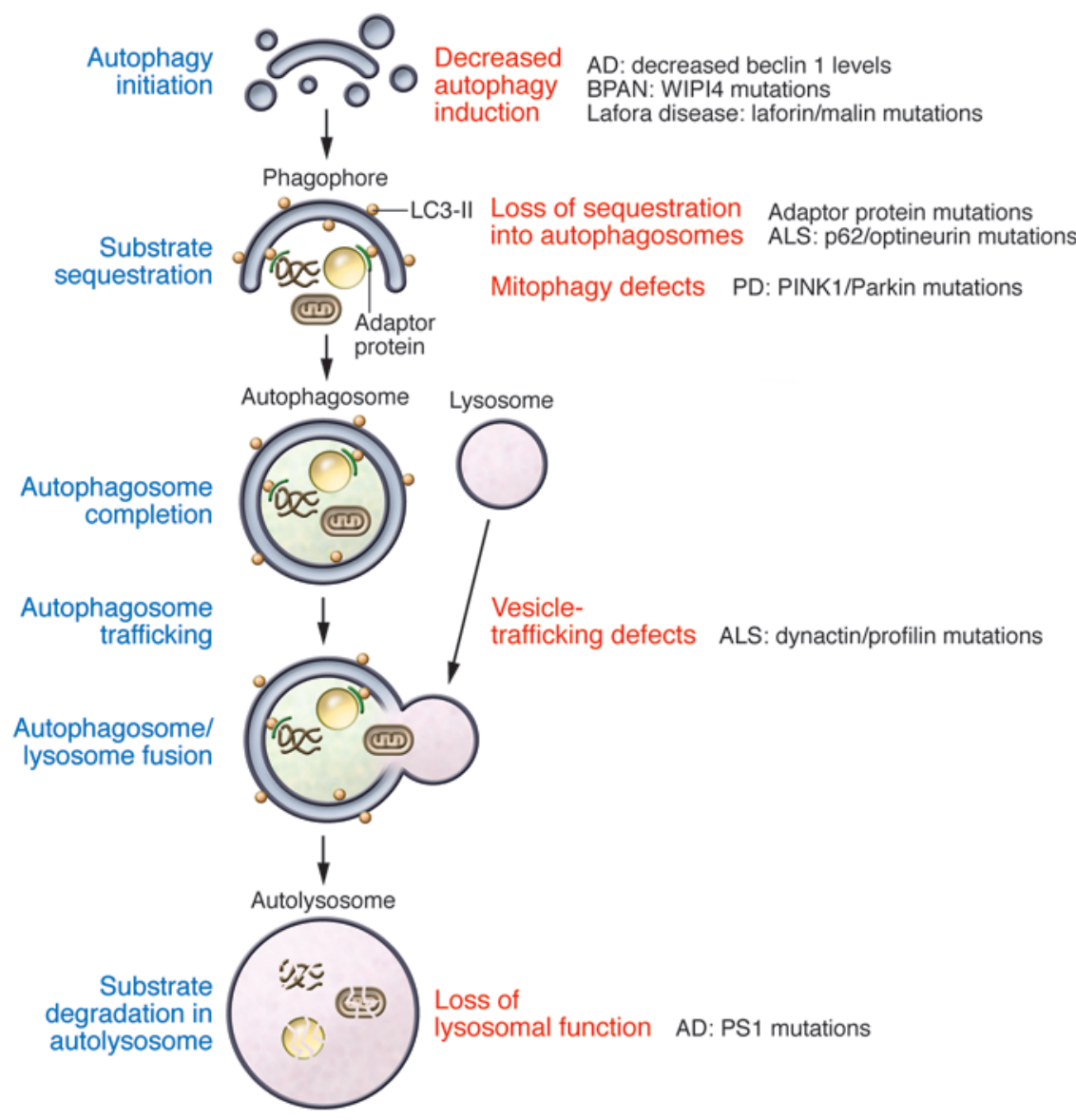

Figure 1. Intersections of the autophagic pathway and neurodegenerative diseases. This schematic shows the progression through the autophagic pathway from formation of the autophagosome to fusion with the lysosome. Red text highlights points of compromise in the pathway that have been demonstrated in neurodegenerative disease, along with examples of causes of this compromise.

$(22,23)$. In contrast, loss of autophagy by conditional knockout of the autophagy protein ATG7 in the forebrains of mice results in phospho-tau accumulation in a pattern similar to a pre-tangle state (24). While deleting tau does not prevent inclusion formation, it does rescue neurodegeneration in these mice (25). Autophagy has been suggested to play a major role in the metabolism of $A \beta$ $(26,27)$; however, it may also be important in the formation of $\mathrm{A} \beta$. The autophagic vesicles that accumulate in AD neurons have been shown to be positive for both APP and PS1 (10, 28). Furthermore, autophagy has been implicated in $A \beta$ secretion, as crossing APP transgenic mice with mice lacking Atg7 in forebrain neurons results in less $A \beta$ extracellular secretion and plaque formation (29). Loss of autophagy may therefore

loss, and lysosome pathology (15). Loss of lysosome acidification, and therefore lysosome function, results in autophagosome accumulation, as autophagosomes do not fuse with dysfunctional lysosomes. Rescue of lysosomal defects can restore autophagic activity. For example cAMP treatment decreased lysosomal $\mathrm{pH}$ in patient fibroblasts (16). Further, deletion of cystatin B (an inhibitor of lysosomal cysteine proteases) in an $\mathrm{AD}$ mouse model enhanced defective lysosomal turnover, promoted $\mathrm{A} \beta$ clearance, and improved mouse cognitive performance (17).

The autophagy gene BECN1, encoding beclin 1, has reduced mRNA levels in AD brain tissue $(18,19)$. Caspase-3, which may be activated in $\mathrm{AD}$ neurons, can also cleave beclin 1, resulting in impaired autophagosome formation (20). When crossed with beclin 1 haploinsufficient mice, mice overexpressing human APP exhibited autophagy disruption and enhanced pathology (18). Because loss of beclin 1 activity reduces autophagosome formation, AD may be associated with defects in both autophagosome biogenesis and autophagosome degradation as a consequence of the lysosomal defects described above.

Beclin 1 disruption may also contribute to $\mathrm{AD}$ in non-neuronal cells. Microglia from $\mathrm{AD}$ patients have reduced beclin 1 and retromer (a key component of the endosomal, protein-sorting machinery) levels, with the beclin 1 disruption further altering correct retromer localization to phagosome membranes and potential receptor-mediated phagocytosis (21).

Tau and $\mathrm{A} \beta$, the two key proteins that aggregate in $\mathrm{AD}$, are autophagy substrates. Induction of autophagy decreases tau levels result in an increase in intracellular $A \beta$ due to both a decrease in clearance and a decrease in secretion of the protein. The role of autophagy in $\mathrm{AD}$ is therefore complex and has been controversial; this may be a function of different effects on autophagy at different stages of the disease as well as the possibility that autophagy may affect different steps of the amyloid life cycle.

$P D$. PD is characterized by the accumulation of $\alpha$-synuclein, whose levels appear central to pathogenesis, as gene duplications of $\alpha$-synuclein are sufficient to cause PD (30). Increased $\alpha$-synuclein levels in cell culture, Drosophila, and mice inhibit autophagy (31) by mislocalizing ATG9, a transmembrane protein with a key function in autophagosome formation. Similar autophagy defects, including ATG9 mislocalization, have recently been observed in cells expressing the VPS35 ${ }^{\mathrm{D} 620 \mathrm{~N}}$ mutation, which causes autosomal-dominant forms of PD (32).

The most common form of autosomal-dominant PD results from mutations in the LRRK2 gene (reviewed in ref. 33). It has been suggested that LRRK2 negatively regulates autophagy, as autophagy is increased following LRRK2 siRNA knockdown or inhibition $(34,35)$. In contrast, overexpression of both wild-type LRRK2 and LRRK2 ${ }^{\text {G2019s }}$, but not catalytically inactive forms of the protein, induces an AMPK-mediated upregulation of autophagy (36). Other studies have reported autophagy upregulation following overexpression of LRRK2 $2^{\mathrm{G} 20195}$ by an ERK1/2-mediated mechanism $(37,38)$.

Perhaps the most prominent link between autophagy and familial forms of PD comes from studies of the roles of PTENinduced putative kinase 1 (PINK1, also known as PARK6) and Par- 
kin RBR E3 ubiquitin protein ligase (PARK2, also known as Parkin) in mitophagy (reviewed in ref. 39). Loss-of-function mutations in Parkin and PINK1 cause autosomal-recessive and sporadic juvenile-onset PD (40-42). These proteins regulate mitophagy, a process whereby damaged mitochondria are degraded. PINK1 associates with damaged mitochondria when membrane potential is lost $(43,44)$, followed by activation of the E3 ubiquitin ligase, Parkin, which ultimately mediates mitophagy. Despite many studies in cell culture with overexpression of these proteins showing effects on mitochondrial clearance, the consequences of loss of Parkin for mitophagy in vivo have been questioned (45). In addition to a role in mitophagy, Parkin has also been suggested to be involved in autophagic clearance of $\alpha$-synuclein, as Parkin overexpression in a rat model system promoted autophagic clearance of toxic proteins including $\alpha$-synuclein (46).

An autosomal-recessive form of Parkinsonism, Kufor-Rakeb syndrome, is caused by mutations in ATPase type 13A2 (ATP13A2, also known as PARK9), which encodes a lysosomal ATPase (47). ATP13A2 regulates lysosomal acidification, which is necessary for autophagosome-lysosome fusion and subsequent substrate degradation, and cells lacking ATP13A2 or patient fibroblasts with ATP13A2 mutations displayed defects in these processes (48)

ALS. ALS is the most common form of motor neuron disease (49). ALS is generally a sporadic disease, but $20 \%$ of cases are familial and have been associated with an increasing number of genes. One of these, sequestosome 1 (SQSTM1), encodes the scaffold protein p62; both point mutations and deletions have been identified in ALS cases $(50,51)$. In addition to its genetic involvement in ALS, p62 is commonly found in aggregates in multiple neurodegenerative diseases, and mutations in SQSTM1 have been identified in Paget's disease of the bone (52). p62 acts as an adaptor to bind ubiquitinated targets to autophagosomeassociated lipidated microtubule-associated light chain 3 (LC3II) for engulfment by autophagosomes (53). While this suggests a clear link between autophagy and ALS, p62 mutations are located throughout the protein, across multiple domains (51), and their effect on autophagy has yet to be fully established. This is also true for ALS-causing mutations in another adaptor, optineurin (54). Optineurin has established roles in xenophagy, a form of autophagy that degrades foreign pathogens (55). Optineurin can also bind to protein aggregates (56) and is sequestered in inclusions in ALS patients as well as in other neurodegenerative diseases $(57,58)$.

The most common known mutation in ALS is an intronic hexanucleotide repeat expansion in the gene C9ORF72. Very little is known about the function of the C9ORF72 protein, and it is currently unclear whether the disease resulting from this mutation is due to a loss of function, gain of function, or both. However preliminary studies on the protein's function suggest that it may have a role in endocytic trafficking and localizes with autophagosomes (59). siRNA knockdown of C9ORF72 increased LC3-II levels, although the study did not fully characterize the meaning of this in terms of autophagy function (59).

Yet another ALS-causing gene with a clear function in autophagy is dynactin 1 (DCTN1) (60). Autophagosomes are formed throughout neurons, and their efficient movement to lysosomerich areas for fusion and cargo degradation is dependent on dynein-mediated transport $(61,62)$. A reduction in this trans- port can result in impaired autophagosome-lysosome fusion, as shown by autophagosome accumulation with enhanced toxicity in cell, Drosophila, and mouse models $(63,64)$. More recently, mutations in DCTN1 have been shown to cause Perry syndrome, a neurodegenerative disease that can present with Parkinsonism and psychiatric changes (65), suggesting a role for autophagic dysfunction in this disease.

HD. HD is caused by polyglutamine repeat expansions in the huntingtin (htt) protein (66). A role for autophagy in HD was first suggested by observations of increased levels of autophagic markers in the brains or tissues of HD patients and in mouse models of the disease (67-69). In HD mouse models, the key autophagic regulator, mTOR, is sequestered into htt aggregates, resulting in an inhibition of signaling, consistent with autophagy induction (70). While this would appear to suggest an upregulation of autophagy in HD, the situation may in fact be more complicated. The positive autophagy regulator, beclin 1 , has also been shown to be sequestered into htt aggregates (71), which would negatively affect autophagosome formation. Moreover, the accumulated autophagosomes observed may not be fully functional; they have been shown to be relatively empty due to inefficient sequestration of cargo, in particular organelles such as mitochondria and lipid droplets, and the increase in autophagosome number is not associated with an increase in protein degradation (72).

In addition, htt itself may control autophagy through a variety of different mechanisms. Two htt-interacting proteins, Rab5 and Rhes, are positive regulators of autophagy $(73,74)$, and htt lacking the polyglutamine repeat region induces autophagy and is protective against toxicity of mutant polyglutamine-expanded htt in cells and mice (75), suggesting a more direct role for htt in autophagy.

While it is not yet clear whether alterations in autophagy are primary factors in the pathogenesis of HD, polymorphisms in the core autophagy gene, $A T G 7$, have been suggested to be associated with earlier age of onset (76).

Hereditary spastic paraplegia. Hereditary spastic paraplegias (HSPs) include a broad group of neurodegenerative diseases involving degeneration associated with the lower extremities. Two forms of HSP have been associated with mutations in genes with a role in autophagy. The first identified was TECPR2, a positive autophagy regulator that interacts with LC3 $(77,78)$. The exact function of this protein is unknown; however, it bears homology to TECPR1, which has been implicated in sequestration of bacteria into autophagosomes, suggesting a role as an autophagy adaptor (77). Type 15 HSP is caused by mutations in ZFYVE26 (79) encoding spastizin (also known as FYVE-CENT), which interacts with the core autophagy protein, beclin 1. Disease-associated mutations in spastizin compromise this interaction, leading to impaired autophagy (79).

Lafora disease. Lafora disease is an autosomal recessive myoclonous epilepsy resulting in early death due to neurodegeneration. Its pathologic hallmark is the accumulation of Lafora bodies (LBs), comprising abnormally hyperphosphorylated polyglucosan with a smaller polyubiquitinated protein component. Most mutations in Lafora disease occur in two proteins, laforin and malin, which form a complex that, when disrupted, drives the disease (reviewed in ref. 80). LB accumulation may be partly the result of altered carbohydrate metabolism (81). However, data from 
patient and animal models clearly support a role for disrupted autophagy in disease onset and progression. Overexpression of laforin induces autophagy, while a reduction in laforin levels has the opposite effect (82). Both laforin- and malin-deficient mice show an early defect in autophagosome biogenesis, which may lead to LB formation (83-85). Both mouse models show reduced levels of autophagosomes and a reduction in total and autophagydependent protein degradation.

Neurodegenerative disorders associated with mutations in core autophagy genes. The likelihood that autophagy defects contribute to neurodegeneration is supported by recent studies that reveal a class of diseases caused by mutations in core autophagy genes. Neurodegeneration with brain iron accumulation 5 (NBIA5; OMIM identifier 300894) is a neurodegenerative disease that presents with movement disorder and intellectual decline. It is currently described as $\beta$-propeller protein-associated neurodegeneration (BPAN) or static encephalopathy of childhood with neurodegeneration in adulthood (SENDA) (86). BPAN is an $\mathrm{X}$-linked dominant disease with loss-of-function mutations identified in the $\beta$-propeller scaffold protein-encoding WDR45/WIPI4, a core autophagy gene that is one of four homologs of yeast Atg18. BPAN appears to be a sporadic disease, with mutations in WIPI4 occurring de novo, as parents and siblings in 20 subjects did not share the mutations (86). In lymphoblastoid cell lines from five patients, reduced autophagic activity and an accumulation of aberrant ATG9A and LC3-II positive autophagic structures were reported (87). This may be a direct result of disrupted ATG9A vesicle translocation at the autophagosome formation site (88) due to altered WIPI4 activity in autophagosome formation $(89,90)$.

Another disease caused by an autophagy gene defect is Vici syndrome (OMIM identifier 242840), a multisystem disorder associated with callosal agenesis. This disorder is caused by recessive mutations in EPG5, an autophagy gene identified first in Caenorhabditis elegans (91). Epg5-null mice develop ALS-like features (92).

\section{Autophagy upregulation as therapy for neurodegenerative disease}

The evidence outlined above suggests a possible role for autophagic dysfunction in the pathogenesis of neurodegenerative diseases, but autophagy also has the ability to decrease the accumulation of toxic, aggregate-prone proteins that cause neurodegeneration. These aggregate-prone proteins are frequently substrates for both the ubiquitin-proteasome system and autophagy as monomers. However, because the oligomeric forms of these proteins cannot pass through the narrow entrance of the proteasome, such higher-order species may be targeted to autophagy. While the appearance of intracytoplasmic inclusions is reduced by autophagy upregulation, our working model is that autophagy does not clear the large aggregates directly, but rather clears the soluble aggregate precursors, shifting the equilibrium from aggregate formation toward degradation (93).

Multiple studies provide proof of principle for the modulation of autophagy as a therapy for neurodegenerative disease. In vitro work examining the effect of autophagy upregulation on the clearance of a wide range of aggregation-prone proteins including those with polyQ and polyA expansions $(5,8)$, mutant tau and ataxin-3 $(8)$, and mutant $\alpha$-synuclein $(6,94)$ has suggested reductions in both intracytoplasmic aggregates and associated cell death. Additionally, autophagy also protects against both proapoptotic (95, 96) and pro-necrotic (97) insults, which may contribute to its benefits. The protective effect seen from autophagy induction in cell models has been successfully translated into a range of animal models, in Drosophila melanogaster models of both tauopathies (8) and HD (70,98), and also in mammalian models of disease, including HD, spinocerebellar ataxia type III, tauopathy, PD, and even familial prion disease (see Table 1 for studies of autophagy upregulation in mouse models of neurodegeneration). Together these studies demonstrate that autophagy upregulation and promotion of aggregation-prone protein degradation ameliorate neurodegenerative pathology.

It is important to note that autophagy inducers are unlikely to be panaceas for all neurodegenerative diseases. Indeed, in diseases associated with impaired autophagosome clearance (e.g., lysosome storage diseases), it is possible that induction of autophagosome biogenesis may be deleterious due to a buildup of autophagosomes that are not cleared effectively. A greater understanding of the potential mechanisms of autophagic dysfunction in neurodegenerative disease as outlined in the first part of this Review is therefore vital for development of therapeutics.

\section{Mechanisms of autophagy upregulation}

The autophagy-modulating agents used in preclinical trials in models of neurodegenerative disease have diverse mechanisms of action. Our understanding of how many of these agents work is not complete; however, they can be divided into mTOR-dependent and mTOR-independent agents.

mTOR-dependent pharmacologic agents. MTOR is found in two different functional complexes. mTOR complex 1 (mTORC1) is a negative autophagy regulator, and ITORC2 is a positive regulator (99). The allosteric mTORC1 inhibitor rapamycin was the first drug to be identified as an autophagy inducer $(100,101)$. Rapamycin forms a complex with FK506-binding protein 12 (FKBP12), which binds to and inhibits the kinase activity of mTORC1 (102, 103). mTORC1 inhibition induces autophagosome formation, as this kinase phosphorylates and inhibits core autophagy proteins such as Unc-51 like autophagy activating kinase 1/2 (ULK1/2) and ATG13 (104-106). Although the mTOR pathway is involved in a wide range of cellular functions (reviewed in ref. 107), the therapeutic effects of rapamycin in models of neurodegenerative disease are predominantly autophagy mediated $(8,98)$. The limited absorption of rapamycin has driven the development of many so-called rapalogs such as temsirolimus (CCI-779), everolimus (RAD001), and ridaforolimus (AP23573). To date, it is these rapalogs that have been investigated most widely in terms of their potential therapeutic value in neurodegenerative diseases. Newly developed mTOR inhibitors include the ATP-competitive mTOR inhibitors (reviewed in ref. 108). Torin1 directly inhibits both mTORC1, including the rapamycin-resistant functions, and mTORC2 (109). Inefficacious concentrations of ATP-competitive mTOR inhibitors can be combined with rapamycin treatment to give complete and selective inhibition of mTORC1, leaving mTORC2 signaling intact (110), which could prove a powerful means of achieving mTOR-dependent autophagy upregulation. This is particularly important, as long-term use of mTORC1/2 
Table 1. Therapeutic upregulation of autophagy in transgenic mouse models of neurodegenerative disease

\section{Transgenic mouse model of neurodegeneration}

HD

\section{HD-N171-82Q (mice express 171 amino acid N-terminal} fragment of htt with 82 glutamines)

PD

D-Line (mice express wild-type human $\alpha$-synuclein)

mThy1 (mice express wild-type human $\alpha$-synuclein)

A53T (mice express A53T human $\alpha$-synuclein in CNS neurons)

AD
Autophagy upregulation agent(s)

Temsirolimus (CCI-779, rapalog), rilmenidine
Aggregate-prone protein cleared $\quad$ Reference $^{A}$

Mutant htt

70,121
CRND8 APP (mice express human APP with the Swedish and Indiana mutations)

20 APP (mice express human APP with Swedish and Indiana mutations)

SwDI APP (mice express human APP with Swedish, Dutch, and lowa mutations)

APP/PS1 (mice express human APP with Swedish mutation, plus human PSEN1 exon 9 deleted)

3xTg-AD (mice express human APP with Swedish mutation, human P301L tau, and M146V PSEN1)

\section{Tauopathy}

$\mathrm{PK}^{-1-} /$ Tau (VLW) (mice express human mutated tau protein with deletion of Parkin)

P3015 tau mice, various lines (express human P3015 tau)

FTLD

FTLD-U (mice overexpress mouse TDP- 43 in the forebrain)

Prion disease

PrP-A116V (mice express mouse prion protein with A116V

mutation and $128 \mathrm{~V}$ polymorphism)

SCA3

Ataxin-3, various lines (mice express human ataxin-3 with an expanded polyglutamine repeat region)
Lentivirus-mediated delivery of beclin 1 , lentivirus-mediated delivery of Atg7 or rapamycin

LDN-57444 (ubiquitin carboxyterminal hydrolase L1 inhibitor)

Nilotinib (tyrosine kinase inhibitor)

\section{Wild-type $\alpha$-synuclein}

Wild-type $\alpha$-synuclein

Mutant $\alpha$-synuclein

114

Anclusion criteria were studies that demonstrated upregulation of autophagy and/or increased aggregate clearance in a transgenic mouse model of neurodegeneration in response to treatment.

catalytic inhibitors impair autophagosome biogenesis (99), raising concerns about the most suitable exposure durations of such drugs in the context of neurodegenerative diseases.

In addition to the drugs that directly inhibit mTOR, other drugs act indirectly through the mTOR pathway. For example, stimulation of the AMPK pathway can upregulate autophagy in an mTOR-dependent manner (111). One example of an AMPK activator with therapeutic potential in neurodegenerative disease is metformin (commonly used in type 2 diabetes) (112). Nilotinib, which has been shown to be protective in multiple mouse models of neurodegeneration through different mechanisms $(113,114)$, can also induce autophagy via AMPK activation (115). Dual class I $\mathrm{PI} 3 \mathrm{~K} / \mathrm{mTORC1}$ inhibitors such as PI103 are potent mTOR-dependent autophagy inducers, which address the negative feedback between mTORC1 and the class I PI3K/AKT pathway (116). However, concerns have been raised that AKT inhibition may increase susceptibility to cell death, rendering class I PI3K/mTORC1 inhib- itors unsuitable as therapy for neurodegenerative disease (117). PI103 is reported not to induce significant apoptosis in cultured cells (118) but is unsuitable for clinical development due to its rapid in vivo metabolism (116).

mTOR-independent pharmacologic agents. mTOR signaling has diverse autophagy-independent functions, including ribosome biogenesis and metabolism (reviewed in ref. 107). Were mTORdependent autophagy induction to be used as long-term therapy for neurodegenerative disease, this would result in non-trivial side effects (immunosuppression and impaired wound healing, for example). Consequently, much work has been dedicated to identifying mTOR-independent upregulators of autophagy. One such study identified several US FDA-approved drugs that ameliorate neurodegenerative pathology in cell, Drosophila, and zebrafish models of HD via mTOR-independent upregulation of autophagy (119). These compounds revealed cyclical regulation of autophagy by the cAMP/EPAC/PLC $\varepsilon / \operatorname{Ins}(1,4,5) \mathrm{P}_{3}$ pathway, together with the 


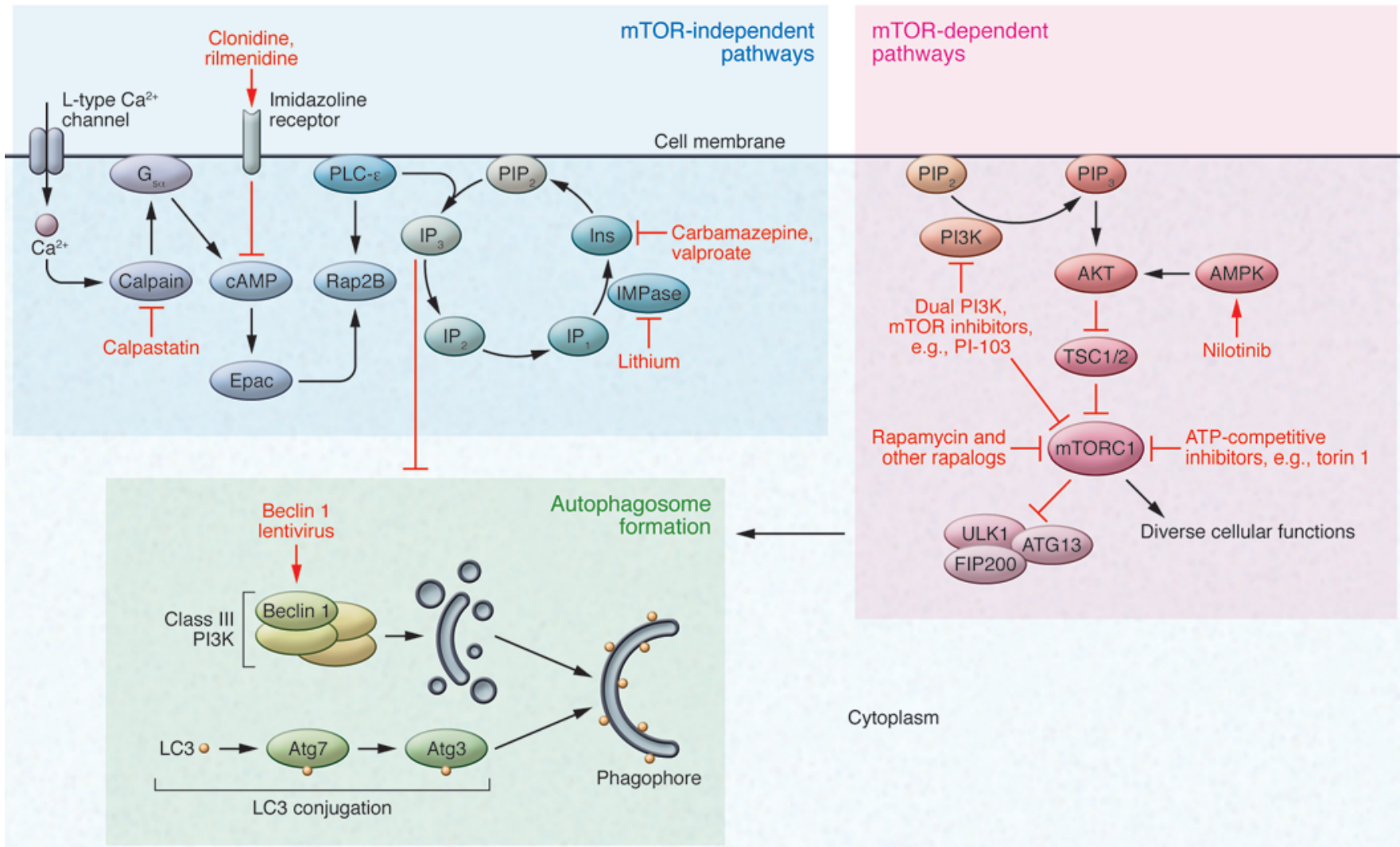

Figure 2. Points of action of autophagy-upregulating agents. This simplified schematic shows some of the pathways known to control autophagy and indicates where drugs and other agents act that are used to upregulate autophagy. In the interest of clarity, not all interactions of these pathways are shown. Both the mTOR-dependent and mTOR-independent pathways negatively regulate the autophagic process, and inhibition of these pathways will lead to an upregulation of autophagy. In the case of mTOR, this may occur through relieving the inhibition of the ULK1 complex, which is a positive regulator of autophagy. In the case of mTOR-independent regulation, the points of interaction between many agents in this category and the autophagy machinery are not known.

$\mathrm{Ca}^{2+} /$ calpain/G $\mathrm{G}_{\mathrm{sa}}$ pathway (see Figure 2 and ref. 119) and showed some overlap with FDA-approved drugs identified in a concurrent screen (120). One of these drugs was the centrally acting antihypertensive rilmenidine, which acts via $G_{i}$-coupled imidazoline receptors (widely distributed in the brain) to reduce cAMP levels (119). Subsequently, rilmenidine was shown to promote clearance of aggregate-prone proteins and improve neurodegenerative pathology in both primary neurons and a transgenic mouse model of HD (121). In light of these results, together with the fact that rilmenidine is safe and suitable for long-term use, a safety trial is ongoing in patients with HD (EudraCT number 2009-018119-14). Other drugs that act through the cAMP/EPAC/PLC $\varepsilon-\operatorname{Ins}(1,4,5) \mathrm{P}_{3}$ pathway include the mood stabilizers lithium (122), carbamazepine, and valproic acid (119). These drugs induce autophagy by reducing Ins $(1,4,5) \mathrm{P}_{3}$ levels via the phosphoinositol cycle; lithium inhibits inositol monophosphatase (122), while carbamazepine and valproic acid inhibit inositol synthesis (119). Subsequent studies have proposed that reducing $\operatorname{Ins}(1,4,5) \mathrm{P}_{3}$ levels might result in less mitochondrial uptake of $\operatorname{Ins}(1,4,5) \mathrm{P}_{3}$ receptor-released $\mathrm{Ca}^{2+}$ and downstream AMPK activation (123), which can occur without mTORC1 inhibition. With regard to the $\mathrm{Ca}^{2+} / \mathrm{calpain} / \mathrm{G}_{\mathrm{sa}}$ pathway, several FDA-approved L-type $\mathrm{Ca}^{2+}$ channel antagonists, including verapamil, nitrendipine, and amiodarone, together with inhibi- tors of calpain activation such as calpeptin and calpastatin, have been found to promote the clearance of aggregation-prone proteins by autophagy (119).

There are also many compounds with therapeutic potential as mTOR-independent autophagy inducers in neurodegenerative disease for which the mechanism of action is under characterized. One example is the disaccharide trehalose, which promotes clearance of mutant htt and mutant $\alpha$-synuclein in mammalian cell culture (124), as well as mutant tau in transgenic mouse models (125-128). Trehalose is neuroprotective in mouse tauopathy models $(125,126)$ and can improve motor and cognitive performance (125). In ALS mouse models, trehalose has been shown to prolong life span $(126,127)$.

\section{Future directions}

Given the therapeutic potential of autophagy upregulation in neurodegenerative disease, there is a clear need to develop more specific autophagy modulators with more tightly defined mechanisms of action. This would enable targeted counteraction of the autophagy deficit present in a particular neurodegenerative condition. However, drug discovery and characterization are slow processes. In the meantime, strategies should be developed to maximize autophagy upregulation with currently available agents, 
while minimizing deleterious side effects. One promising approach is the simultaneous induction of autophagy by mTOR-dependent and mTOR-independent pathways. As a proof of principle, combined rapamycin and lithium treatment in a Drosophila model of HD induced autophagy and reduced neurodegenerative pathology to a greater extent than either drug alone (129). Additionally, combined rapamycin and trehalose treatment exerts an additive effect on the clearance of mutant htt and mutant $\alpha$-synuclein by autophagy (124). In addition to small molecules, autophagy upregulation may be amenable with peptide strategies, which may have promise in neurodegeneration (130).

It is also important to be mindful of other therapies for neurodegenerative disease that might impede the clearance of pathogenic aggregation-prone proteins by autophagy. For instance, antioxidants have been used to counteract the increased oxidative stress seen in many neurodegenerative diseases, but some classes of antioxidants are reported to counteract the beneficial effects of autophagy induction in Drosophila and zebrafish models of HD (131).
In conclusion, therapeutic strategies aimed at upregulation of autophagy appear promising. As our knowledge of the pathways controlling this process increases, we are likely to be able to develop ever more selective therapies that will upregulate autophagy in a manner that will be optimal for the specific disease to be treated.

\section{Acknowledgments}

We are grateful for support from a Wellcome Trust Principal Research Fellowship (to D.C. Rubinsztein), a Wellcome Trust/ MRC Strategic Grant on Neurodegeneration (to D.C. Rubinsztein), a Sims Scholarship from the FEE Fund and JB Fund (to R.A. Frake), the NIHR Biomedical Research Centre at Addenbrooke's Hospital, the Tau Consortium, and Alzheimer's Research UK.

Address correspondence to: David C. Rubinsztein, University of Cambridge, Cambridge Institute for Medical Research, Addenbrooke's Hospital, Hills Road, Cambridge, CB2 2XY, United Kingdom. Phone: 44.1223.762608; E-mail: dcr1000@cam.ac.uk.
1. Rubinsztein DC, Shpilka T, Elazar Z. Mechanisms of autophagosome biogenesis. Curr Biol. 2012;22(1):R29-R34.

2. Komatsu M, et al. Loss of autophagy in the central nervous system causes neurodegeneration in mice. Nature. 2006;441(7095):880-884.

3. Hara T, et al. Suppression of basal autophagy in neural cells causes neurodegenerative disease in mice. Nature. 2006;441(7095):885-889.

4. Komatsu M, et al. Essential role for autophagy protein Atg7 in the maintenance of axonal homeostasis and the prevention of axonal degeneration. Proc Natl Acad Sci U S A. 2007;104(36):14489-14494.

5. Ravikumar B, Duden R, Rubinsztein D. Aggregate-prone proteins with polyglutamine and polyalanine expansions are degraded by autophagy. Hum Mol Genet. 2002;11(9):1107-1117.

6. Webb JL, Ravikumar B, Atkins J, Skepper JN, Rubinsztein DC. Alpha-Synuclein is degraded by both autophagy and the proteasome. J Biol Chem. 2003;278(27):25009-25013.

7. Barmada SJ, et al. Autophagy induction enhances TDP43 turnover and survival in neuronal ALS models. Nat Chem Biol. 2014;10(8):677-685.

8. Berger Z, et al. Rapamycin alleviates toxicity of different aggregate-prone proteins. $\mathrm{Hum} \mathrm{Mol}$ Genet. 2006;15(3):433-442.

9. Nixon R, et al. Extensive involvement of autophagy in Alzheimer disease: an immuno-electron microscopy study. J Neuropathol Exp Neurol. 2005;64(2):113-122.

10. Boland B, et al. Autophagy induction and autophagosome clearance in neurons: relationship to autophagic pathology in Alzheimer's disease. J Neurosci. 2008;28(27):6926-6937.

11. Lee JH, et al. Lysosomal proteolysis and autophagy require presenilin 1 and are disrupted by Alzheimer-related PS1 mutations. Cell. 2010;141(7):1146-1158.

12. Sherrington $\mathrm{R}$, et al. Cloning of a gene bearing missense mutations in early-onset familial Alzheimer's disease. Nature. 1995;375(6534):754-760.

13. Levy-Lahad E, et al. Candidate gene for the chro- mosome 1 familial Alzheimer's disease locus. Science. 1995;269(5226):973-977.

14. Goate A, et al. Segregation of a missense mutation in the amyloid precursor protein gene with familial Alzheimer's disease. Nature. 1991;349(6311):704-706.

15. Cataldo AM, et al. Presenilin mutations in familial Alzheimer disease and transgenic mouse models accelerate neuronal lysosomal pathology. J Neuropathol Exp Neurol. 2004;63(8):821-830.

16. Coffey EE, Beckel JM, Laties AM, Mitchell CH. Lysosomal alkalization and dysfunction in human fibroblasts with the Alzheimer's disease-linked presenilin $1 \mathrm{~A} 246 \mathrm{E}$ mutation can be reversed with cAMP. Neuroscience. 2014;263:11-124.

17. Yang DS, et al. Reversal of autophagy dysfunction in the TgCRND8 mouse model of Alzheimer's disease ameliorates amyloid pathologies and memory deficits. Brain. 2011;134(pt 1):258-277.

18. Pickford F, et al. The autophagy-related protein beclin 1 shows reduced expression in early Alzheimer disease and regulates amyloid beta accumulation in mice. JClin Invest. 2008;118(6):2190-2199.

19. Small S, et al. Model-guided microarray implicates the retromer complex in Alzheimer's disease. Ann Neurol. 2005;58(6):909-919.

20. Rohn TT, Wirawan E, Brown RJ, Harris JR, Masliah E, Vandenabeele P. Depletion of Beclin-1 due to proteolytic cleavage by caspases in the Alzheimer's disease brain. Neurobiol Dis. 2011;43(1):68-78.

21. Lucin KM, et al. Microglial beclin 1 regulates retromer trafficking and phagocytosis and is impaired in Alzheimer's disease. Neuron. 2013;79(5):873-886.

22. Berger $Z$, et al. Deleterious and protective properties of an aggregate-prone protein with a polyalanine expansion. Hum Mol Genet. 2006;15(3):453-465.

23. Kruger U, Wang Y, Kumar S, Mandelkow EM. Autophagic degradation of tau in primary neurons and its enhancement by trehalose. Neurobiol Aging. 2012;33(10):2291-2305.
24. Luna-Munoz J, Chavez-Macias L, Garcia-Sierra F, Mena R. Earliest stages of tau conformational changes are related to the appearance of a sequence of specific phospho-dependent tau epitopes in Alzheimer's disease. J Alzheimers Dis. 2007;12(4):365-375.

25. Inoue K, et al. Macroautophagy deficiency mediates age-dependent neurodegeneration through a phospho-tau pathway. Mol Neurodegener. 2012;7:48.

26. Vingtdeux V, Chandakkar P, Zhao H, d'Abramo C, Davies P, Marambaud P. Novel synthetic small-molecule activators of AMPK as enhancers of autophagy and amyloid- $\beta$ peptide degradation. FASEB J. 2011;25(1):219-231.

27. Tian Y, Bustos V, Flajolet M, Greengard P. A small-molecule enhancer of autophagy decreases levels of $A \beta$ and APP-CTF via Atg5-dependent autophagy pathway. FASEB J. 2011;25(6):1934-1942.

28. Yu W, et al. Macroautophagy - a novel $\beta$-amyloid peptide-generating pathway activated in Alzheimer's disease. JCell Biol. 2005;171(1):87-98.

29. Nilsson $P$, et al. A $\beta$ secretion and plaque formation depend on autophagy. Cell Rep. 2013;5(1):61-69.

30. Singleton AB, et al. $\alpha$-Synuclein locus triplication causes Parkinson's disease. Science. 2003;302(5646):841.

31. Winslow AR, et al. $\alpha$-Synuclein impairs macroautophagy: implications for Parkinson's disease. JCell Biol. 2010;190(6):1023-1037.

32. Zavodszky E, et al. Mutation in VPS35 associated with Parkinson's disease impairs WASH complex association and inhibits autophagy. Nat Commun. 2014;5:3828.

33. Singleton AB, Farrer MJ, Bonifati V. The genetics of Parkinson's disease: progress and therapeutic implications. Mov Disord. 2013;28(1):14-23.

34. Alegre-Abarrategui J, et al. LRRK2 regulates autophagic activity and localizes to specific membrane microdomains in a novel human genomic reporter cellular model. Hum Mol Genet. 2009;18(21):4022-4034.

35. Manzoni C, et al. Inhibition of LRRK2 kinase 
activity stimulates macroautophagy. Biochim Biophys Acta. 2013;1833(12):2900-2910.

36. Gomez-Suaga P, et al. Leucine-rich repeat kinase 2 regulates autophagy through a calcium-dependent pathway involving NAADP. Hum Mol Genet. 2012;21(3):511-525.

37. Bravo-San Pedro JM, et al. The LRRK2 G2019S mutant exacerbates basal autophagy through activation of the MEK/ERK pathway. Cell Mol Life Sci. 2013;70(1):121-136.

38. Plowey ED, Cherra SJ, Cherra SJ 3rd, Liu YJ, Chu CT. Role of autophagy in G2019S-LRRK2-associated neurite shortening in differentiated SH-SY5Y cells. J Neurochem. 2008;105(3):1048-1056.

39. de Vries RL, Przedborski S. Mitophagy and Parkinson's disease: be eaten to stay healthy. Mol Cell Neurosci. 2013;55:37-43.

40. Kitada T, et al. Mutations in the parkin gene cause autosomal recessive juvenile parkinsonism. Nature. 1998;392(6676):605-608.

41. Valente EM, et al. Localization of a novel locus for autosomal recessive early-onset parkinsonism, PARK6, on human chromosome 1p35-p36. Am J Hum Genet. 2001;68(4):895-900.

42. Valente EM, et al. Hereditary early-onset Parkinson's disease caused by mutations in PINK1. Science. 2004;304(5674):1158-1160.

43. Narendra D, Tanaka A, Suen D, Youle R. Parkin is recruited selectively to impaired mitochondria and promotes their autophagy.J Cell Biol. 2008;183(5):795-803.

44. Matsuda N, et al. PINK1 stabilized by mitochondrial depolarization recruits Parkin to damaged mitochondria and activates latent Parkin for mitophagy. JCell Biol. 2010;189(2):211-221.

45. Sterky FH, Lee S, Wibom R, Olson L, Larsson NG. Impaired mitochondrial transport and Parkin-independent degeneration of respiratory chain-deficient dopamine neurons in vivo. Proc Natl Acad Sci U S A. 2011;108(31):12937-12942.

46. Lonskaya I, Hebron ML, Algarzae NK, Desforges $\mathrm{N}$, Moussa CE. Decreased parkin solubility is associated with impairment of autophagy in the nigrostriatum of sporadic Parkinson's disease. Neuroscience. 2012;232:90-105.

47. Ramirez A, et al. Hereditary parkinsonism with dementia is caused by mutations in ATP13A2, encoding a lysosomal type 5 P-type ATPase. Nat Genet. 2006;38(10):1184-1191.

48. Dehay B, et al. Loss of P-type ATPase ATP13A2/ PARK9 function induces general lysosomal deficiency and leads to Parkinson disease neurodegeneration. Proc Natl Acad Sci U S A. 2012;109(24):9611-9616.

49. Baumer D, Talbot K, Turner MR. Advances in motor neurone disease. JR Soc Med. 2014;107(1):14-21.

50. Hirano $\mathrm{M}$, et al. Mutations in the gene encoding p62 in Japanese patients with amyotrophic lateral sclerosis. Neurology. 2013;80(5):458-463.

51. Teyssou E, et al. Mutations in SQSTM1 encoding p62 in amyotrophic lateral sclerosis: genetics and neuropathology. Acta Neuropathol. 2013;125(4):511-522.

52. Laurin N, Brown JP, Morissette J, Raymond V. Recurrent mutation of the gene encoding sequestosome 1 (SQSTM1/p62) in Paget disease of bone. Am J Hum Genet. 2002;70(6):1582-1588.
53. Matsumoto G, Wada K, Okuno M, Kurosawa $M$, Nukina N. Serine 403 phosphorylation of p62/SQSTM1 regulates selective autophagic clearance of ubiquitinated proteins. Mol Cell. 2011;44(2):279-289.

54. Maruyama $\mathrm{H}$, et al. Mutations of optineurin in amyotrophic lateral sclerosis. Nature. 2010;465(7295):223-226.

55. Wild P, et al. Phosphorylation of the autophagy receptor optineurin restricts Salmonella growth. Science. 2011;333(6039):228-233.

56. Korac J, et al. Ubiquitin-independent function of optineurin in autophagic clearance of protein aggregates. JCell Sci. 2013;126(pt 2):580-592.

57. Schwab C, Yu S, McGeer EG, McGeer PL. Optineurin in Huntington's disease intranuclear inclusions. Neurosci Lett. 2012;506(1):149-154.

58. Mori F, et al. Optineurin immunoreactivity in neuronal nuclear inclusions of polyglutamine diseases (Huntington's, DRPLA, SCA2, SCA3) and intranuclear inclusion body disease. Acta Neuropathol. 2012;123(5):747-749.

59. Farg MA, et al. C9ORF72, implicated in amytrophic lateral sclerosis and frontotemporal dementia, regulates endosomal trafficking. Hum Mol Genet. 2014;23(13):3579-3595.

60. Puls I, et al. Mutant dynactin in motor neuron disease. Nat Genet. 2003;33(4):455-456.

61. Jahreiss L, Menzies FM, Rubinsztein DC. The itinerary of autophagosomes: from peripheral formation to kiss-and-run fusion with lysosomes. Traffic. 2008;9(4):574-587.

62. Kimura S, Noda T, Yoshimori T. Dynein-dependent movement of autophagosomes mediates efficient encounters with lysosomes. Cell Struct Funct. 2008;33(1):109-122.

63. Ravikumar B, et al. Dynein mutations impair autophagic clearance of aggregate-prone proteins. Nat Genet. 2005;37(7):771-776.

64. Yamamoto M, Suzuki SO, Himeno M. The effects of dynein inhibition on the autophagic pathway in glioma cells. Neuropathology. 2010;30(1):1-6.

65. Farrer MJ, et al. DCTN1 mutations in Perry syndrome. Nat Genet. 2009;41(2):163-165.

66. [No authors listed]. A novel gene containing a trinucleotide repeat that is expanded and unstable on Huntington's disease chromosomes. The Huntington's Disease Collaborative Research Group. Cell. 1993;72(6):971-983.

67. Heng MY, et al. Early autophagic response in a novel knock-in model of Huntington disease. Hum Mol Genet. 2010;19(19):3702-3720.

68. Nagata E, Sawa A, Ross CA, Snyder SH. Autophagosome-like vacuole formation in Huntington's disease lymphoblasts. Neuroreport. 2004;15(8):1325-1328.

69. Rudnicki D, Pletnikova O, Vonsattel J, Ross C, Margolis R. A comparison of huntington disease and huntington disease-like 2 neuropathology. J Neuropathol Exp Neurol. 2008;67(4):366-374.

70. Ravikumar B, et al. Inhibition of mTOR induces autophagy and reduces toxicity of polyglutamine expansions in fly and mouse models of Huntington disease. Nat Genet. 2004;36(6):585-595.

71. Shibata M, et al. Regulation of intracellular accumulation of mutant Huntingtin by Beclin 1.J Biol Chem. 2006;281(20):14474-14485.

72. Martinez-Vicente $\mathrm{M}$, et al. Cargo recognition failure is responsible for inefficient autophagy in Huntington's disease. Nat Neurosci. 2010;13(5):567-576.

73. Ravikumar B, Imarisio S, Sarkar S, O'Kane C, Rubinsztein D. Rab5 modulates aggregation and toxicity of mutant huntingtin through macroautophagy in cell and fly models of Huntington disease. JCell Sci. 2008;121(pt 10):1649-1660.

74. Mealer RG, Murray AJ, Shahani N, Subramaniam S, Snyder SH. Rhes, a striatal-selective protein implicated in Huntington disease, binds beclin-1 and activates autophagy. J Biol Chem. 2014;289(6):3547-3554.

75. Zheng S, Clabough EB, Sarkar S, Futter M, Rubinsztein DC, Zeitlin SO. Deletion of the huntingtin polyglutamine stretch enhances neuronal autophagy and longevity in mice. PLoS Genet. 2010;6(2):e1000838

76. Metzger S, et al. Age at onset in Huntington's disease is modified by the autophagy pathway: implication of the V471A polymorphism in Atg7. Hum Genet. 2010;128(4):453-459.

77. Oz-Levi D, et al. Mutation in TECPR2 reveals a role for autophagy in hereditary spastic paraparesis. Am J Hum Genet. 2012;91(6):1065-1072.

78. Behrends C, Sowa ME, Gygi SP, Harper JW. Network organization of the human autophagy system. Nature. 2010;466(7302):68-76.

79. Vantaggiato $C$, et al. Defective autophagy in spastizin mutated patients with hereditary spastic paraparesis type 15. Brain. 2013;136(pt 10):3119-3139.

80. Roma-Mateo C, Sanz P, Gentry MS. Deciphering the role of malin in the lafora progressive myoclonus epilepsy. IUBMB Life. 2012;64(10):801-808.

81. Delgado-Escueta AV. Advances in lafora progressive myoclonus epilepsy. Curr Neurol Neurosci Rep. 2007;7(5):428-433.

82. Aguado C, et al. Laforin, the most common protein mutated in Lafora disease, regulates autophagy. Hum Mol Genet. 2010;19(14):2867-2876.

83. Puri R, Suzuki T, Yamakawa K, Ganesh S. Hyperphosphorylation and aggregation of Tau in laforin-deficient mice, an animal model for Lafora disease. J Biol Chem. 2009;284(34):22657-22663.

84. Tagliabracci VS, et al. Laforin is a glycogen phosphatase, deficiency of which leads to elevated phosphorylation of glycogen in vivo. Proc Natl Acad Sci U S A. 2007;104(49):19262-19266.

85. Criado O, et al. Lafora bodies and neurological defects in malin-deficient mice correlate with impaired autophagy. Hum Mol Genet. 2012;21(7):1521-1533.

86. Haack TB, et al. Exome sequencing reveals de novo WDR45 mutations causing a phenotypically distinct, X-linked dominant form of NBIA. Am J Hum Genet. 2012;91(6):1144-1149.

87. Saitsu H, et al. De novo mutations in the autophagy gene WDR45 cause static encephalopathy of childhood with neurodegeneration in adulthood. Nat Genet. 2013;45(4):445-449.

88. Lu Q, et al. The WD40 repeat PtdIns(3)P-binding protein EPG- 6 regulates progression of omegasomes to autophagosomes. Dev Cell. 2011;21(2):343-357.

89. Itakura E, Kishi-Itakura C, Koyama-Honda I, Mizushima N. Structures containing Atg9A and the ULK1 complex independently target depolarized mitochondria at initial stages of Parkin-mediated 
mitophagy.JCell Sci. 2012;125(pt 6):1488-1499.

90. Orsi A, et al. Dynamic and transient interactions of Atg9 with autophagosomes, but not membrane integration, are required for autophagy. Mol Biol Cell. 2012;23(10):1860-1873.

91. Cullup T, et al. Recessive mutations in EPG5 cause Vici syndrome, a multisystem disorder with defective autophagy. Nat Genet. 2013;45(1):83-87.

92. Zhao H, et al. Mice deficient in Epg5 exhibit selective neuronal vulnerability to degeneration. JCell Biol. 2013;200(6):731-741.

93. Rubinsztein DC. The roles of intracellular protein-degradation pathways in neurodegeneration. Nature. 2006;443(7113):780-786.

94. Spencer B, et al. Beclin 1 gene transfer activates autophagy and ameliorates the neurodegenerative pathology in alpha-synuclein models of Parkinson's and Lewy body diseases. J Neurosci. 2009;29(43):13578-13588.

95. Ravikumar B, Berger Z, Vacher C, O'Kane CJ, Rubinsztein DC. Rapamycin pre-treatment protects against apoptosis. Hum Mol Genet. 2006;15(7):1209-1216.

96. Boya P, et al. Inhibition of macroautophagy triggers apoptosis. Mol Cell Biol. 2005;25(3):1025-1040.

97. Wu YT, et al. Autophagy plays a protective role during zVAD-induced necrotic cell death. Autophagy. 2008;4(4):457-466.

98. Wang T, Lao U, Edgar BA. TOR-mediated autophagy regulates cell death in Drosophila neurodegenerative disease. JCell Biol. 2009;186(5):703-711.

99. Renna M, et al. IGF-1 receptor antagonism inhibits autophagy. Hum Mol Genet. 2013;22(22):4528-4544.

100.Blommaart EF, Luiken JJ, Blommaart PJ, van Woerkom GM, Meijer AJ. Phosphorylation of ribosomal protein $\mathrm{S} 6$ is inhibitory for autophagy in isolated rat hepatocytes. J Biol Chem. 1995;270(5):2320-2326.

101. Noda T, Ohsumi Y. Tor, a phosphatidylinositol kinase homologue, controls autophagy in yeast. JBiol Chem. 1998;273(7):3963-3966.

102. Cardenas ME, Heitman J. FKBP12-rapamycin target TOR2 is a vacuolar protein with an associated phosphatidylinositol-4 kinase activity. EMBO J. 1995;14(23):5892-5907.

103. Lorenz MC, Heitman J. TOR mutations confer rapamycin resistance by preventing interaction with FKBP12-rapamycin. J Biol Chem. 1995;270(46):27531-27537.

104.Ganley IG, Lam du H, Wang J, Ding X, Chen S, Jiang X. ULK1.ATG13.FIP200 complex mediates mTOR signaling and is essential for autophagy. J Biol Chem. 2009;284(18):12297-12305.

105. Hosokawa N, et al. Nutrient-dependent mTORC1 association with the ULK1-Atg13FIP200 complex required for autophagy. Mol Biol Cell. 2009;20(7):1981-1991.

106.Jung CH, et al. ULK-Atg13-FIP200 complexes mediate $\mathrm{mTOR}$ signaling to the autophagy machinery. Mol Biol Cell. 2009;20(7):1992-2003.

107.Zoncu R, Efeyan A, Sabatini DM. mTOR: from growth signal integration to cancer, diabetes and ageing. Nat Rev Mol Cell Biol. 2011;12(1):21-35.

108. Benjamin D, Colombi M, Moroni C, Hall MN. Rapamycin passes the torch: a new genera- tion of mTOR inhibitors. Nat Rev Drug Discov. 2011;10(11):868-880.

109. Thoreen CC, et al. An ATP-competitive mammalian target of rapamycin inhibitor reveals rapamycin-resistant functions of mTORC1. J Biol Chem. 2009;284(12):8023-8032.

110. Nyfeler B, et al. Relieving autophagy and 4EBP1 from rapamycin resistance. Mol Cell Biol. 2011;31(14):2867-2876.

111. Meley D, et al. AMP-activated protein kinase and the regulation of autophagic proteolysis. J Biol Chem. 2006;281(46):34870-34879.

112. Buzzai M, et al. Systemic treatment with the antidiabetic drug metformin selectively impairs p53-deficient tumor cell growth. Cancer Res. 2007;67(14):6745-6752.

113. Lonskaya I, Hebron ML, Desforges NM, Schachter JB, Moussa CE. Nilotinib-induced autophagic changes increase endogenous parkin level and ubiquitination, leading to amyloid clearance. J Mol Med (Berl). 2014;92(4):373-386.

114. Hebron ML, Lonskaya I, Moussa CE. Nilotinib reverses loss of dopamine neurons and improves motor behavior via autophagic degradation of $\alpha$-synuclein in Parkinson's disease models. Hum Mol Genet. 2013;22(16):3315-3328.

115. Yu HC, Lin CS, Tai WT, Liu CY, Shiau CW, Chen KF. Nilotinib induces autophagy in hepatocellular carcinoma through AMPK activation. J Biol Chem. 2013;288(25):18249-18259.

116. Raynaud FI, et al. Pharmacologic characterization of a potent inhibitor of class I phosphatidylinositide 3-kinases. Cancer Res. 2007;67(12):5840-5850.

117. Rubinsztein DC, Codogno P, Levine B. Autophagy modulation as a potential therapeutic target for diverse diseases. Nat Rev Drug Discov. 2012;11(9):709-730.

118. Degtyarev M, et al. Akt inhibition promotes autophagy and sensitizes PTEN-null tumors to lysosomotropic agents. JCell Biol. 2008;183(1):101-116.

119. Williams A, et al. Novel targets for Huntington's disease in an mTOR-independent autophagy pathway. Nat Chem Biol. 2008;4(5):295-305.

120.Zhang L, et al. Small molecule regulators of autophagy identified by an image-based high-throughput screen. Proc Natl Acad Sci U S A. 2007;104(48):19023-19028.

121. Rose $\mathrm{C}$, et al. Rilmenidine attenuates toxicity of polyglutamine expansions in a mouse model of Huntington's disease. Hum Mol Genet. 2010;19(11):2144-2153.

122. Sarkar S, et al. Lithium induces autophagy by inhibiting inositol monophosphatase. JCell Biol. 2005;170(7):1101-1111.

123. Cardenas $C$, et al. Essential regulation of cell bioenergetics by constitutive InsP3 receptor $\mathrm{Ca}^{2+}$ transfer to mitochondria. Cell.2010;142(2):270-283.

124.Sarkar S, Davies J, Huang Z, Tunnacliffe A, Rubinsztein D. Trehalose, a novel mTOR-independent autophagy enhancer, accelerates the clearance of mutant huntingtin and $\alpha$-synuclein. JBiol Chem. 2007;282(8):5641-5652.

125. Rodriguez-Navarro JA, et al. Trehalose ameliorates dopaminergic and tau pathology in parkin deleted/tau overexpressing mice through autophagy activation. Neurobiol Dis.
2010;39(3):423-438.

126. Schaeffer V, Lavenir I, Ozcelik S, Tolnay M, Winkler DT, Goedert M. Stimulation of autophagy reduces neurodegeneration in a mouse model of human tauopathy. Brain. 2012;135(pt 7):2169-2177.

127. Castillo K, et al. Trehalose delays the progression of amyotrophic lateral sclerosis by enhancing autophagy in motoneurons. Autophagy. 2013;9(9):1308-1320.

128.Zhang X, et al. MTOR-independent, autophagic enhancer trehalose prolongs motor neuron survival and ameliorates the autophagic flux defect in a mouse model of amyotrophic lateral sclerosis. Autophagy. 2014;10(4):588-602.

129.Sarkar S, Krishna G, Imarisio S, Saiki S, O’Kane CJ, Rubinsztein DC. A rational mechanism for combination treatment of Huntington's disease using lithium and rapamycin. Hum Mol Genet. 2008;17(2):170-178.

130. Shoji-Kawata S, et al. Identification of a candidate therapeutic autophagy-inducing peptide. Nature. 2013;494(7436):201-206.

131. Underwood BR, et al. Antioxidants can inhibit basal autophagy and enhance neurodegeneration in models of polyglutamine disease. Hum Mol Genet. 2010;19(17):3413-3429.

132. Crews $\mathrm{L}$, et al. Selective molecular alterations in the autophagy pathway in patients with Lewy body disease and in models of alpha-synucleinopathy. PLoS One. 2010;5(2):e9313.

133. Cartier AE, et al. Differential effects of UCHL1 modulation on alpha-synuclein in PD-like models of alpha-synucleinopathy. PLoS One. 2012;7(4):e34713.

134. Steele JW, et al. Latrepirdine improves cognition and arrests progression of neuropathology in an Alzheimer's mouse model. Mol Psychiatry. 2013;18(8):889-897.

135. Spilman P, et al. Inhibition of mTOR by rapamycin abolishes cognitive deficits and reduces amyloid- $\beta$ levels in a mouse model of Alzheimer's disease. PLoS One. 2010;5(4):e9979.

136.Vingtdeux $\mathrm{V}$, et al. AMP-activated protein kinase signaling activation by resveratrol modulates amyloid- $\beta$ peptide metabolism. J Biol Chem. 2010;285(12):9100-9113.

137. Jiang $\mathrm{T}$, et al. Temsirolimus promotes autophagic clearance of amyloid- $\beta$ and provides protective effects in cellular and animal models of Alzheimer's disease. Pharmacol Res. 2014;81:54-63.

138. Zhu Z, et al. Arctigenin effectively ameliorates memory impairment in Alzheimer's disease model mice targeting both $\beta$-amyloid production and clearance. J Neurosci. 2013;33(32):13138-13149.

139. Li L, et al. Autophagy enhancer carbamazepine alleviates memory deficits and cerebral amyloid- $\beta$ pathology in a mouse model of Alzheimer's disease. Curr Alzheimer Res. 2013;10(4):433-441.

140.Caccamo A, Majumder S, Richardson A, Strong $\mathrm{R}$, Oddo S. Molecular interplay between mammalian target of rapamycin (mTOR), amyloid- $\beta$, and Tau: effects on cognitive impairments. J Biol Chem. 2010;285(17):13107-13120.

141. Chu C, et al. Induction of autophagy by a novel small molecule improves abeta pathology and ameliorates cognitive deficits. PLoS One. 2013;8(6):e65367. 
142. Majumder S, Richardson A, Strong R, Oddo S. Inducing autophagy by rapamycin before, but not after, the formation of plaques and tangles ameliorates cognitive deficits. PLoS One. 2011;6(9):e25416.

143. Shimada K, et al. Long-term oral lithium treatment attenuates motor disturbance in tauopathy model mice: implications of autophagy promotion. Neurobiol Dis. 2012;46(1):101-108.

144.Schaeffer V, Goedert M. Stimulation of autophagy is neuroprotective in a mouse model of human tauopathy. Autophagy. 2012;8(11):1686-1687.

145. Congdon EE, et al. Methylthioninium chloride (methylene blue) induces autophagy and attenuates tauopathy in vitro and in vivo. Autophagy. 2012;8(4):609-622.

146.Caccamo A, et al. mTOR regulates tau phosphorylation and degradation: implications for Alzheimer's disease and other tauopathies. Aging Cell. 2013;12(3):370-380.

147. Wang IF, et al. Autophagy activators rescue and alleviate pathogenesis of a mouse model with proteinopathies of the TAR DNA-binding protein 43. Proc Natl Acad Sci US A. 2012;109(37):15024-15029.

148.Cortes CJ, Qin K, Cook J, Solanki A, Mastrianni
JA. Rapamycin delays disease onset and prevents PrP plaque deposition in a mouse model of Gerstmann-Straussler-Scheinker disease. J Neurosci. 2012;32(36):12396-12405.

149. Menzies FM, Huebener J, Renna M, Bonin M, Riess O, Rubinsztein DC. Autophagy induction reduces mutant ataxin-3 levels and toxicity in a mouse model of spinocerebellar ataxia type 3 . Brain. 2010;133(pt 1):93-104.

150. Silva-Fernandes A, et al. Chronic treatment with 17-DMAG improves balance and coordination in a new mouse model of Machado-Joseph disease. Neurotherapeutics. 2014;11(2):433-449. 